\title{
Towards Discovery of Information Granules
}

\author{
Andrzej Skowron ${ }^{1} \&$ Jaroslaw Stepaniuk ${ }^{2}$ \\ 1 Institute of Mathematics, University of Warsaw, \\ Banacha 2, 02-097 Warsaw, Poland, \\ E-mail: skowron@mimuw.edu.pl \\ 2 Institute of Computer Science, Bialystok University of Technology, \\ Wiejska 45A, 15-351 Bialystok, Poland, \\ E-mail: jstepan@ii.pb.bialystok.pl
}

\begin{abstract}
The amount of electronic data available is growing very fast and this explosive growth in databases has generated a need for new techniques and tools that can intelligently and automatically extract implicit, previously unknown, hidden and potentially useful information and knowledge from these data. Such tools and techniques are the subject of the field of Knowledge Discovery in Databases. Information granulation is a very natural concept, and appears (under different names) in many methods related to e.g. data compression, divide and conquer, interval computations, neighborhood systems, and rough sets among others. In this paper we discuss information granulation in knowledge discovery. The notions related to information granules are their syntax and semantics as well as the inclusion and closeness (similarity) of granules. We discuss some problems of KDD assuming knowledge is represented in the form of information granules. In particular we use information granules to deal with the problem of stable (robust) patterns extraction.
\end{abstract}

\section{Introduction}

Knowledge Discovery in Databases (or KDD for short) has been defined as the nontrivial extraction of implicit, previously unknown, and potentially useful knowledge from data [3. It uses machine learning, statistical and visualization techniques, rough set theory and other techniques to discovery and present knowledge in a form which is easily comprehensible to humans.

Notions of granule [12], [13], [9], [7], [10] and similarity (inclusion or closeness) of granules are very natural in knowledge discovery. The necessity to consider similarity of granules instead of their equality is a consequence of uncertainty under which granules are processed.

The aim of the paper is to show that the association rules 22 can be treated as simple examples of relations on information granules. The left and right hand sides of association rules [2] describe granules and support and confidence specify degrees of the granule inclusion represented by the formula on the left hand side of association rule into the granule represented by the formula on the right hand side of association rule. 
We generalize a simple notion of granules represented by attribute value vectors as well as closeness (inclusion) relation to the case of hierarchical granules of concepts. We claim that in KDD there is a need for algorithmic methods to discover much more complex relations between complex information granules. We discuss examples of information granules and we consider two kinds of basic relations between them, namely (rough) inclusion and closeness. The relations between more complex information granules can be defined by extension of the relations defined on parts of the information granules.

\section{Granule Inclusion and Closeness}

One of the main direction of research for successful construction of intelligent systems is to develop a calculus of information granules [12, [13, [7]. We emphasize the fact that granules and their closeness are important for KDD.

In this section we will present several examples of granules starting from the simplest ones. A general scheme of defining more complex granule from simpler ones can be explored using rough mereological approach [6]. We are adding here a new aspect of construction of more complex granules from simpler ones and the problem of extension of rough inclusion and closeness of granules on these complex granules. Closeness between granules $G, G^{\prime}$ of degree at least $p$ will be denoted by $c l_{p}\left(G, G^{\prime}\right)$ (or $c l\left(G, G^{\prime}\right) \geq p$ ). Similarly, inclusion between granules $G, G^{\prime}$ of degree at least $p$ will be denoted by $\nu_{p}\left(G, G^{\prime}\right)\left(\right.$ or $\left.\nu\left(G, G^{\prime}\right) \geq p\right)$. $c l$ and $\nu$ are functions with values in the interval $[0,1]$ of real numbers $\left(\right.$ or $\left.[0,1]^{k}\right)$.

A general scheme for construction of hierarchical granules and their closeness can be described recursively using the following metarule: if granules of order $\leq k$ and their closeness have been defined then the closeness $c l_{p}\left(G, G^{\prime}\right)$ (at least in degree $p$ ) between granules $G, G^{\prime}$ of order $k+1$ can be defined by applying an appropriate operator $F$ to closeness of components of $G, G^{\prime}$, respectively.

\subsection{Elementary Granules - Conjunctions of Selectors}

The simplest case of granules can be defined using information system $I S=$ $(U, A)$. In this case elementary information granules are defined by $E F_{B}(x)$, where $E F_{B}$ is a conjunction of selectors of the form $a=a(x), B \subseteq A$ and $x \in U$. Let $G_{I S}=\left\{E F_{B}(x): B \subseteq A \& x \in U\right\}$. In the standard rough set model [5] elementary granules describe indiscernibility classes with respect to some subsets of attributes. In a more general setting see e.g. [8], 11] tolerance (similarity) classes are described. The crisp inclusion of $\alpha$ in $\beta$, where $\alpha, \beta \in\left\{E F_{B}(x): B \subseteq A \& x \in U\right\}$ is defined by $\|\alpha\|_{I S} \subseteq\|\beta\|_{I S}$, where $\|\alpha\|_{I S}$ and $\|\beta\|_{I S}$ are sets of objects from IS satisfying $\alpha$ and $\beta$, respectively. The noncrisp inclusion, known in KDD, for the case of association rules is defined by two thresholds $t$ and $t^{\prime}$ and the following conditions

$\operatorname{supp}_{I S}(\alpha, \beta)=\|\alpha \wedge \beta\|_{I S} \geq t$, and $\operatorname{conf}_{I S}(\alpha, \beta)=\frac{\operatorname{supp}_{I S}(\alpha, \beta)}{\|\alpha\|_{I S}} \geq t^{\prime}$.

Granules inclusion can be defined using different schemes e.g. by $\nu_{t, t^{\prime}}^{I S}(\alpha, \beta)$ if and only if $\operatorname{supp}_{I S}(\alpha, \beta) \geq t \& \operatorname{conf} f_{I S}(\alpha, \beta) \geq t^{\prime}$ or 
$\nu_{t}^{I S}(\alpha, \beta)$ if and only if $\operatorname{conf}_{I S}(\alpha, \beta) \geq t$. In the latter case one can define closeness of the granules by

$c l_{t}^{I S}(\alpha, \beta)=\min \left\{t^{\prime}, t^{\prime \prime}\right\}$, where $\nu_{t^{\prime}}^{I S}(\alpha, \beta)$ and $\nu_{t^{\prime \prime}}^{I S}(\beta, \alpha)$ hold.

\subsection{Decision Rules as Granules}

One can define granules corresponding to rules if $\alpha$ then $\beta$ as pairs $(\alpha, \beta)$ of elementary information granules together with information about their closeness of components measured e.g. using confidence coefficients (see e.g. [7]). Having such granules $r=(\alpha, \beta), r^{\prime}=\left(\alpha^{\prime}, \beta^{\prime}\right)$ with degrees of closeness at least $t, t^{\prime}$, respectively one can define closeness of $r$ and $r^{\prime}$. This definition should satisfy the following natural condition: if $r$ and $r^{\prime}$ are sufficiently close (in a given information system $I S$ ) and $\alpha, \beta$ are close at least in degree $t$ (in $I S$ ) then $\alpha^{\prime}$ and $\beta^{\prime}$ are close in degree $t^{\prime}$ (in $I S$ ) where $\left|t-t^{\prime}\right|$ is "sufficiently small". One simple attempt to define such closeness can be as follows: $r$ and $r^{\prime}$ are close in degree at least $t$ if and only if $\alpha, \alpha^{\prime}$ are close in degree at least $t$ (in $I S$ ) and $\beta, \beta^{\prime}$ are close in degree at least $t$ (in $I S$ ).

Another way of defining inclusion of granules is as follows

$\nu^{I S}\left((\alpha, \beta),\left(\alpha^{\prime}, \beta^{\prime}\right)\right)=w_{1} \bullet \operatorname{con} f_{I S}\left(\alpha, \alpha^{\prime}\right)+w_{2} \bullet \operatorname{con} f_{I S}\left(\beta, \beta^{\prime}\right)$, where $w_{1}+$ $w_{2}=1$ and $w_{1}, w_{2} \geq 0$ are some weights to be tuned for a given data.

\subsection{Sets of Rules}

An important problem related to association rules is that the number of such rules generated even from simple data table can be large. Hence, one should search for methods of aggregating close association rules. We suggest that this can be defined as searching for some close information granules.

Let us consider two finite sets of association rules $R$ and $R^{\prime}$. One can treat them as higher order information granules. These new granules $R, R^{\prime}$ can be treated as close in a degree at least $t$ (in $I S$ ) if and only if there exists a relation rel between rules $R$ and $R^{\prime}$ such that:

1. for any $r \in R$ there is $r^{\prime} \in R^{\prime}$ such that $\left(r, r^{\prime}\right) \in r e l$ and $r$ is close to $r^{\prime}$ (in $I S)$ in degree at least $t$.

2. for any $r^{\prime} \in R^{\prime}$ there is $r \in R$ such that $\left(r, r^{\prime}\right) \in r e l$ and $r$ is close to $r^{\prime}$ (in $I S)$ in degree at least $t$.

One can consider a searching problem for a granule $R^{\prime}$ of minimal size such that $R$ and $R^{\prime}$ are close (see e.g. [1]).

Another way of defining closeness of two granules $G_{1}, G_{2}$ represented by sets of rules can be described as follows.

For example if the granule $G_{1}$ consists of rules: if $\alpha_{1}$ then $d=1$, if $\alpha_{2}$ then $d=1$, if $\alpha_{3}$ then $d=1$, if $\beta_{1}$ then $d=0$, if $\beta_{2}$ then $d=0$ and the granule $G_{2}$ consists of rules: if $\gamma_{1}$ then $d=1$, if $\gamma_{2}$ then $d=0$, then $c l\left(G_{1}, G_{2}\right)=\min \left\{c l\left(G_{1}^{1}, \gamma_{1}\right), c l\left(G_{1}^{2}, \gamma_{2}\right)\right\}$, where $G_{1}^{1}$ is the granule $\alpha_{1} \vee \alpha_{2} \vee \alpha_{3}$ and $G_{1}^{2}$ is the granule $\beta_{1} \vee \beta_{2}$. 


\subsection{Power Sets of Granules}

Let $G$ be a set of granules and let $\varphi$ be a property of sets of granules from $G$ (e.g. $\varphi(X)$ if and only if $X$ is a tolerance class of a given tolerance $\tau \subseteq G \times G)$. Then $P_{\varphi}(G)=\{X \subseteq G: \varphi(X)$ holds $\}$. Closeness of granules $X, Y \in P_{\varphi}(G)$ can be defined by

$c l_{t}(X, Y)$ if and only if $\inf _{g \in X, g^{\prime} \in Y} c l_{t}\left(g, g^{\prime}\right)$.

Let us observe that the closeness definition can depend on application. For example instead of the above definition one can use the following $c l_{t}(X, Y)$ if and only if there exists $r \subseteq X \times Y$ such that

1. for any $x \in X$ there is $y \in Y$ such that $(x, y) \in r$ and $x, y$ are close in degree at least $t$.

2. for any $y \in Y$ there is $x \in X$ such that $(x, y) \in r$ and $x, y$ are close in degree at least $t$.

The stability of granules $g, g^{\prime} \in G$ with respect to $P_{\varphi}(G)$ is defined by $g, g^{\prime}$ are $P_{\varphi}(G)$ stable in degree at least $t$ if and only if

$\forall_{X, Y \in P_{\varphi}(G)}\left(g \in X \& g^{\prime} \in Y \rightarrow c l_{t}\left(g, g^{\prime}\right)\right)$.

Extracting stable patterns from data is an important task in KDD.

\subsection{Stability of Granules}

We consider granules specified by parameterized formulas $\alpha(p), \beta(p)$ together with parameterized closeness relation $c l_{q}(\bullet, \bullet)$ between sets of objects representing the semantics of these granules. The parameter $q \in[0,1]$ is representing the degree of closeness. We assume that values of parameter $p$ are points in the metric space with metric $\rho$. Hence the intended meaning of $c l_{q}(\alpha(p), \beta(p))$ is that granules $\alpha(p), \beta(p)$ (parameterized by $p$ ) are close at least in the degree $q$. Let us assume that $\varepsilon>0$ is a given stability threshold and let $c l_{q_{0}}\left(\alpha\left(p_{0}\right), \beta\left(p_{0}\right)\right)$ holds for some $p_{0}, q_{0}$. We would like to check if there exists $\delta>\delta_{0}\left(\delta_{0}\right.$ is a given threshold) such that for any $p$

if $\rho\left(p_{0}, p\right) \leq \delta$ then $c l_{q}(\alpha(p), \beta(p))$ for some $q$ such that $q \geq q_{0}-\varepsilon$.

The above condition is specifying that granules $\alpha\left(p_{0}\right), \beta\left(p_{0}\right)$ are not only close in degree $q$ but their closeness is also stable with respect to changes of parameter $p$.

Example 1. Let us assume that the rule

if $a \in[1,1.5) \wedge b \in[-1,0)$ then $d=1$

is an association rule in a given decision table $D T=(U, A \cup\{d\})$ with coefficients

$\operatorname{supp}_{D T}(a \in[1,1.5) \wedge b \in[-1,0), d=1)=0.6 * \operatorname{card}(U)$,

$\operatorname{conf}_{D T}(a \in[1,1.5) \wedge b \in[-1,0), d=1)=0.7$

Let $q_{0}=(0.7,0.6), p_{0}=(0,0)$ and $\varepsilon=0.1$. We ask if there exists $\delta>\delta_{0}=$ 0.01 such that for any $p=\left(p_{1}, p_{2}\right)$ if $\sqrt{p_{1}^{2}+p_{2}^{2}} \leq \delta$ then the rule

if $a \in\left[1-p_{1}, 1.5+p_{1}\right) \wedge b \in\left[-1-p_{2}, p_{2}\right)$ then $d=1$

is true in a given decision table $D T$ with coefficients 


$$
\begin{aligned}
& \operatorname{supp}_{1}=\operatorname{supp}_{D T}\left(a \in\left[1-p_{1}, 1.5+p_{1}\right) \wedge b \in\left[-1-p_{2}, p_{2}\right), d=1\right), \\
& \operatorname{conf}_{1}=\operatorname{con}_{D T}\left(a \in\left[1-p_{1}, 1.5+p_{1}\right) \wedge b \in\left[-1-p_{2}, p_{2}\right), d=1\right), \\
& \text { such that } \sqrt{\left(\frac{\operatorname{supp} p_{1}}{\operatorname{card}(U)}-0.6\right)^{2}+\left(\operatorname{con} f_{1}-0.7\right)^{2}} \leq \varepsilon .
\end{aligned}
$$

\subsection{Dynamic Granules}

For a given decision table $D T$ and information vector $\alpha$ one can define a subtable $D T_{\alpha}$ of $D T$ consisting of all objects from $D T$ with information vectors being extension of $\alpha$. For any such table one can generate the set of decision rules $\operatorname{Rule}\left(D T_{\alpha}\right)$. In this way we obtain a new crisp information granules $\left(\alpha, \operatorname{Rule}\left(D T_{\alpha}\right)\right)$. Let us assume that on information vectors a tolerance $\tau$ is defined. Hence we obtain more complex information granules denoted by $\left(\tau(\alpha),\left\{\operatorname{Rule}\left(D T_{\beta}\right)\right\}_{\beta \in \tau(\alpha)}\right)$, where $\tau(\alpha)=\{\beta:(\alpha, \beta) \in \tau\}$. They can be treated as soft information granules.

Obviously sets of rules from $\left\{\operatorname{Rule}\left(D T_{\beta}\right)\right\}_{\beta \in \tau(\alpha)}$ should be sufficiently close if one would like to represent such granules using their representatives e.g. taking an arbitrary $\beta$ from $\tau(\alpha)$ and Rule $\left(D T_{\beta}\right)$. An interesting problem arises to search for a compact representation of such soft granules relative to $\tau$.

Let us consider one more example. The sentence "if the situation specified by evidence $\alpha$ the algorithm Rule $\left(D T_{\alpha}\right)$ is performed and when a new evidence $\beta$ appears the algorithm Rule $\left(D T_{\alpha \wedge \beta}\right)$ is performed" can be represented by the crisp granule

$$
G(\alpha, \beta, D T)=\left(\left(\alpha, \operatorname{Rule}\left(D T_{\alpha}\right)\right),\left(\alpha \wedge \beta, \text { Rule }\left(D T_{\alpha \wedge \beta}\right)\right)\right) .
$$

The soft granules $G(\alpha, \beta, \tau, D T)$ relative to a given tolerance $\tau$ can be represented by the family of all granules $G\left(\alpha^{\prime}, \beta^{\prime}, D T\right)$ such that $\alpha^{\prime} \in \tau(\alpha)$ and $\beta^{\prime} \in \tau(\beta)$. Assuming the closeness of granules of the form $G(\alpha, \beta, D T)$ has been defined one can define the closeness of $G(\alpha, \beta, D T)$ and $G(\alpha, \beta, \tau, D T)$ by taking infimum of closeness degrees between $G(\alpha, \beta, D T)$ and components of $G(\alpha, \beta, \tau, D T)$. Hence if $G(\alpha, \beta, D T)$ and $G(\alpha, \beta, \tau, D T)$ are close at least in degree $t$ it means that the crisp granule $G(\alpha, \beta, D T)$ can represent the soft granule $G(\alpha, \beta, \tau, D T)$ at least in the degree $t$.

\section{Conclusions}

Our approach can be treated as a step towards understanding of complex information granules and their role in KDD. We have pointed out that information granules and their closeness (inclusion) are basic tools for data mining and knowledge discovery. Some higher order patterns important for KDD have been described by means of higher order granules. We also suggest that the optimization of information granules can be performed by tuning up parameters in an optimization process searching for robust granules. In our next papers we show algorithmic methods for extracting from data patterns described by the discussed above higher order granules. 


\section{Acknowledgments}

This research was supported by the grant No. 8 T11C 02315 from the State Committee for Scientific Research, the Bialystok University of Technology Rector's Grant W/II/3/98 and Research Program of the European Union - ESPRITCRIT 2 No. 20288.

\section{References}

1. Ågotnes T.: Filtering Large Propositional Rule Sets While Retaining Classifier Performance, NTNU Research Report 1999.

2. Agrawal R., Mannila H., Srikant R., Toivonen H., Verkano A.: Fast Discovery of Association Rules, Fayyad U.M., Piatetsky-Shapiro G., Smyth P., Uthurusamy R. (eds.): Advances in Knowledge Discovery and Data Mining, The AAAI Press/The MIT Press 1996, pp. 307-328.

3. Fayyad U.M., Piatetsky-Shapiro G., Smyth P., Uthurusamy R. (eds.): Advances in Knowledge Discovery and Data Mining, The AAAI Press/The MIT Press 1996.

4. Lin T.Y.: Granular Computing on Binary Relations I Data Mining and Neighborhood Systems, L. Polkowski, A. Skowron (eds.), Rough Sets in Knowledge Discovery 1. Methodology and Applications, Physica-Verlag, Heidelberg, 1998, pp. 107-121.

5. Pawlak Z.: Rough Sets. Theoretical Aspects of Reasoning about Data, Kluwer Academic Publishers, Dordrecht, 1991.

6. Polkowski, L., Skowron, A.: Rough Mereology: A New Paradigm for Approximate Reasoning, International Journal of Approximate Reasoning, Vol. 15, No 4, 1996, pp. 333-365.

7. Polkowski L., Skowron A.: Towards Adaptive Calculus of Granules, Proceedings of the FUZZ-IEEE'98 International Conference, Anchorage, Alaska, USA, May 5-9, 1998.

8. Skowron A., Stepaniuk J.: Tolerance Approximation Spaces, Fundamenta Informaticae, Vol. 27, 1996, pp. 245-253.

9. Skowron A., Stepaniuk J.: Constructive Information Granules, Proceedings of the 15th IMACS World Congress on Scientific Computation, Modelling and Applied Mathematics, Berlin, Germany, August 24-29, 1997. Artificial Intelligence and Computer Science 4, 1997, pp. 625-630.

10. Skowron A., Stepaniuk J.: Information Granules and Approximation Spaces, Proceedings of the 7th International Conference on Information Processing and Management of Uncertainty in Knowledge-based Systems, IPMU'98, Paris, France, July 6-10, 1998, pp. 1354-1361.

11. Stepaniuk J.: Approximation Spaces, Reducts and Representatives, L. Polkowski, A. Skowron (eds.), Rough Sets in Knowledge Discovery 2. Applications, Case Studies and Software Systems, Physica-Verlag, Heidelberg, 1998, pp. 109-126.

12. Zadeh L.A.: Fuzzy Logic = Computing with Words, IEEE Trans. on Fuzzy Systems Vol. 4, 1996, pp. 103-111.

13. Zadeh L.A.: Toward a Theory of Fuzzy Information Granulation and Its Certainty in Human Reasoning and Fuzzy Logic, Fuzzy Sets and Systems Vol. 90, 1997, pp. 111-127. 